\title{
ANÁLISE DOS PERIÓDICOS QUALIS/CAPES: VISÃO GERAL DA ÁREA DE ENSINO EM CIÊNCIAS E MATEMÁTICA
}

\section{ANALYSIS OF QUALIS/CAPES PERIODICALS: OVERVIEW OF THE AREA IN OF SCIENCE AND MATHEMATICS EDUCATION}

\author{
Saulo C. Seiffert Santos ${ }^{1}$ \\ Alessandra Crystian Engles dos Reis ${ }^{2}$ \\ Cléria Maria Wendling ${ }^{3}$ \\ Kassiana da Silva Miguel ${ }^{4}$ \\ Luciana Del Castanhel Peron ${ }^{5}$ \\ Maira Vanessa Bär ${ }^{6}$ \\ Wander Mateus Branco Meier ${ }^{7}$ \\ Márcia Borin da Cunha ${ }^{8}$
}

\begin{abstract}
Resumo: No Brasil, a Coordenação de Aperfeiçoamento de Pessoal de Nível Superior (CAPES) propõe à produção científica, avaliação da qualidade dos periódicos, por meio de um sistema denominado Qualis. Buscamos descrever os periódicos da área de Ensino na especificidade de Educação/Ensino em Ciências e/ou Matemática, presente na edição Qualis CAPES (2013-2016). A partir do estudo dos periódicos A1 a B1 da área de Ensino. Aferimos 506 títulos, desconsiderando duplicidades na plataforma Sucupira. A Análise foi qualitativa e descritiva com uso do Formulário Google. Os dados pesquisados foram relacionados à fundação, disponibilização pela internet, bases de indexadores e citações. Encontramos um quadro de 82 revistas com escopo voltado a área de Ensino de Ciências e Matemática. Do total de
\end{abstract}

${ }^{1}$ Doutorando do Programa de Pós-Graduação em Educação em Ciências e Educação Matemática (PPGECEM) da Universidade Estadual do Oeste do Paraná (Unioeste), Cascavel, Paraná, Brasil. E-mail: sauloseiffert@ufam.edu.br

${ }^{2}$ Doutoranda do Programa de Pós-Graduação em Educação em Ciências e Educação Matemática (PPGECEM) da Universidade Estadual do Oeste do Paraná (Unioeste), Cascavel, Paraná, Brasil. E-mail: acereis75@gmail.com

3 Doutoranda do Programa de Pós-Graduação em Educação em Ciências e Educação Matemática (PPGECEM) da Universidade Estadual do Oeste do Paraná (Unioeste), Cascavel, Paraná, Brasil. E-mail: cleriamwe@gmail.com

4 Doutoranda do Programa de Pós-Graduação em Educação em Ciências e Educação Matemática (PPGECEM) da Universidade Estadual do Oeste do Paraná (Unioeste), Cascavel, Paraná, Brasil. E-mail: kassianamiguelunioeste@gmail.com

5 Doutoranda do Programa de Pós-Graduação em Educação em Ciências e Educação Matemática (PPGECEM) da Universidade Estadual do Oeste do Paraná (Unioeste), Cascavel, Paraná, Brasil. E-mail: lucianaperon@hotmail.com

6 Doutoranda do Programa de Pós-Graduação em Educação em Ciências e Educação Matemática (PPGECEM) da Universidade Estadual do Oeste do Paraná (Unioeste), Cascavel, Paraná, Brasil. E-mail: mairabio4@hotmail.com

7 Doutorando do Programa de Pós-Graduação em Educação em Ciências e Educação Matemática (PPGECEM) da Universidade Estadual do Oeste do Paraná (Unioeste), Cascavel, Paraná, Brasil. E-mail: wandermateus@yahoo.com.br

${ }^{8}$ Docente do Programa de Pós-Graduação em Educação em Ciências e Educação Matemática (PPGECEM) da Universidade Estadual do Oeste do Paraná (Unioeste), Cascavel, Paraná, Brasil. E-mail: borin.unioeste@gmail.com 
periódicos, 46 em Ciências, 27 em Matemática e 9 periódicos Mistos. Os dados foram organizados conforme a caracterização das revistas. Realizamos análise e discussão, e por fim sugerimos recomendações sobre a qualidade.

Palavras-chave: Periódicos; Ensino de Ciências; Educação Matemática; Qualis.

\begin{abstract}
In Brazil, the Coordination of Improvement of Higher Education Personnel (CAPES in portuguese) proposes to the scientific production, evaluation of the quality of journals, through a system called Qualis. We seek to describe the journals of the Teaching area in the specificity of Education / Teaching in Science and / or Mathematics, present in the edition Qualis CAPES (2013-2016). From the study of the periodicals A1 to B1 of the Teaching area. We checked 506 titles, disregarding duplications on the Sucupira Platform. Qualitative and descriptive analysis using the Google Form. The data surveyed were related to the foundation, availability by intenet, bases of indexers and citations. We found a table of 82 journals with scope focused on the area ofScience and Mathematics Teaching. Of the total periodicals, 46 in Science, 27 in Mathematics and 9 in Mixed Periodicals. The data were organization according to the characterization of the journals. We carry out analysis and discussion, and finally we suggest recommendations on quality.
\end{abstract}

Keywords: Periodicals; Science Education; Mathematical Education; Qualis.

\title{
1 Introdução
}

A comunicação e a publicação de resultados da ciência são atividades intrínsecas da atividade científica. A publicização dos resultados entre a comunidade ocorre preferencialmente via periódicos que, segundo Malozze (1999), teriam como função, registro oficial e público da ciência; meio de divulgação; prestigiar cientistas; alertar sobre as pesquisas e seus resultados junto à comunidade; arquivar, divulgar e promover padrões de qualidade.

A publicação da “[...] comunicação científica, por sua vez, diz respeito à transferência de informações científicas, tecnológicas ou associadas a inovações e que se destinam aos especialistas em determinadas áreas do conhecimento" (BUENO, 2010, p. 2). E nesta missão, a pesquisa brasileira tem se esmerado na organização sistemática por parte de órgãos governamentais brasileiros para a melhoria da quantidade e qualidade das investigações.

No Brasil, a Coordenação de Aperfeiçoamento de Pessoal de Nível Superior (CAPES) lançou em novembro do ano 2000 o Programa de Apoio à Aquisição de Periódicos (PAAP), em parceria com o Ministério da Educação e Cultura (MEC), para suprir o déficit das bibliotecas para pesquisas em Instituições de Nível Superior, organizando também, no Portal da CAPES, um acervo de pesquisa.

Atualmente o Portal da CAPES conta com acervo de 38 mil títulos completos, 134 bases de referenciais e 11 bases dedicadas a patentes. Nassi-Calò (2014) informa que o Portal da CAPES recebeu uma receita totalmente pública junto ao Conselho 
Nacional de Desenvolvimento Científico e Tecnológico (CNPq) de 3 milhões de dólares, divididos entre os 200 melhores periódicos no ano de 2014.

A CAPES, no processo de avaliação dos programas de pós-graduação, segundo Nassi-Calò (2014, on-line) e Barata (2016), avalia a produção a partir da qualidade dos periódicos pelo sistema Qualis, em um ranqueamento por níveis A1, A2, B1, B2, B3, B4, B5 e C e pode ser acessado pela Plataforma Sucupira (on-line). Nisto, a posição dos periódicos no Qualis determina seu prestígio e influência para a quantidade e qualidade dos trabalhos submetidos (FERREIRA; KRZYZANOWSKI, 2003).

Para Machado e Jesus (2012), a introdução de avaliações externas, a padronização dos periódicos e a avaliação dos pesquisadores pela "régua" da quantidade de produção resultou em um excesso de confiança nesses critérios, gerando uma atenção ao cumprimento das regras formais e práticas que atendem às normas, mas que não mantém o vínculo com a busca de qualidade de fato. Para as autoras, “[...] os limites da indução produtivista da CAPES que produz quantidade, de duvidosa qualidade e não coíbe desvirtuações (sic) da finalidade da ciência" (MACHADO; JESUS, 2012, p. 5), é um problema a ser considerado.

Diante desse quadro, neste artigo temos como proposta fazer uma descrição qualitativa a respeito do foco metodológico de trabalhos publicados recentemente em periódicos da área de Ensino nas especificidades declarada de Educação e/ou Ensino em Ciências e/ou/com a Educação Matemática, presente na edição Qualis CAPES (20132016). Entendendo que na área atual de Ensino se encontram reunidos todos os periódicos de ensino de diversas áreas do conhecimento.

Ressaltamos que obtivemos a listagem dos periódicos no Portal Sucupira e, fizemos busca dos periódicos em site de busca "Google". Como também as comparações de métrica em citação foram realizadas no Google Acadêmico.

\section{Narrativa}

Este artigo é produto oriundo da disciplina "Análise e Produção de Artigos em Educação em Ciências e Educação Matemática", ofertada pelo Programa de PósGraduação em Educação em Ciências e Educação Matemática, da Universidade Estadual do Oeste do Paraná - Unioeste, no segundo semestre de 2017. Sua motivação surgiu de discussões realizadas no âmbito da disciplina em torno da qualidade das produções científicas e dos processos de avaliação propostos pela CAPES para 
padronizar e impulsionar a produtividade da pesquisa brasileira. Com o intuito de proceder uma avaliação dos periódicos da área consideramos a necessidade de realizar um levantamento amplo dos periódicos de Ensino.

A partir do estudo da quantidade de periódicos na plataforma Sucupira, decidimos analisar aqueles estratificados pelo ranqueamento da CAPES como excelente ou de alta qualidade, ou seja, Qualis A1, A2 e B1, no quadriênio 2013-2016. Nessa busca inicial encontramos 710 periódicos, havendo títulos repetidos decorrente da divulgação em meio digital e impresso. A partir da correção dessa discrepância restaram 506 periódicos sobre os quais coletamos diversas informações relativas ao impacto, as normas e ao conteúdo, conforme as informações disponibilizadas no site da revista, construindo, deste modo, um acervo quantitativo desses periódicos.

Em função da grande quantidade de dados e, considerando a diversidade da formação dos autores (Biologia, Matemática, Ciências, Enfermagem e Pedagogia) foram selecionados periódicos cujo foco e escopo indicam a área de Ensino de Ciências e de Educação Matemática ${ }^{9}$. Diante dessas condições, identificamos 46 periódicos da área de Ciências, 27 periódicos na área de Matemática e 9 periódicos Mistos (de Ciências e Matemática) presentes na Tabela 1. A relação completa dos periódicos selecionados está no Apêndice A.

Tabela 1: Periódicos de Ensino em Ciências, Matemática e Misto do Qualis A1, A2 e B1 (2013/2016)

\begin{tabular}{ccc} 
Periódicos & $\mathbf{N}$ & Percentagem \\
Ciências & 46 & $56,0 \%$ \\
Matemática & 27 & $33,3 \%$ \\
Misto & 9 & $10,7 \%$ \\
Total & 82 & $100,0 \%$ \\
\hline
\end{tabular}

Fonte: Os autores.

Uma vez definido o corpus de análise elaboramos um formulário on-line pelo Google Drive (Formulário Google), no qual cada autor preencheu os dados referentes à revista que estava analisando. Essa ferramenta contribui para realização de um trabalho colaborativo no que se refere à contagem absoluta e relativa, categorização, correção de seções, ente outros.

\footnotetext{
${ }^{9}$ No escopo procuramos os termos Educação em Ciências, Ensino de Ciências, Ensino de Matemática e Educação Matemática. Outros termos semelhantes não foram selecionados. É possível que periódicos que publicam nestas áreas não tenham sido selecionados devido ao critério adotado.
} 
A partir do momento em que os dados da planilha on-line foram analisados foi possível a organização de tabelas e categorização dos artigos. Essa etapa produziu um rico diálogo entre os pesquisadores, no sentido de compreender a situação das publicações mais recentes destes periódicos.

\section{Resultados}

Verificamos um quadro de 82 revistas periódicos, sendo 31 periódicos de origem internacional e 51 periódicos nacionais. Sendo estas revistas fundadas e publicadas online predominantemente a partir do ano 2000, normalmente (na sua maioria) com mais de dois indexadores, e de periodicidade quadrimestrais e semestrais.

Nas próximas seções vamos apresentar os dados de categorização, tempo de publicação, relação entre revistas nacionais e internacionais.

\subsection{Qualis}

A coleta se concentrou, como já dissemos, nas revistas Qualis A1, A2 e B1 em ensino de ciências em que no escopo apresenta-se claramente o vínculo de pesquisa na área de ensino/educação em ciências e/ou ensino/educação matemática. Assim organizamos em categorias os periódicos (ciência, matemática e ciência \& matemática), conforme a tabela 2 .

Tabela 2: Periódicos de Ensino em Ciências, Matemática e Misto do Qualis A1, A2 e B1 (2013/2016)

\begin{tabular}{ccccc}
\hline Qualis & Ciências & Matemática & Ciê. \& Mat. & Total \\
A1 & 7 & 6 & 0 & 13 \\
A2 & 12 & 10 & 6 & 28 \\
B1 & 27 & 11 & 3 & 41 \\
Total & $\mathbf{4 6}$ & $\mathbf{2 7}$ & $\mathbf{9}$ & $\mathbf{8 2}$ \\
\hline
\end{tabular}

Fonte: Os autores.

Encontramos mais periódicos na área de Ciências do que de Matemática, e algumas revistas que no seu escopo informou que trabalhava com as duas áreas. Observamos que no nível A1 há um equilíbrio entre a quantidade de periódicos de Ciências e Matemática, nos demais estratos predominam os de Ciências. 
O número maior de revistas em Ciências do que em Matemática pode ter como pressuposto que as Ciências abrangem a Biologia, a Física e a Química, o que concentra um maior número de pesquisadores e consequentemente maior número de publicações.

Percebemos que há uma diferença de quase 10 títulos em quantidade de periódicos A2 para B1, em este último ultrapassa 40 títulos. Um avanço em relação à edição anterior do Qualis que havia um número muito abreviado em relação a edição atual. Esse aspecto expressa um avanço da própria área de Ensino.

\subsection{Fundação das revistas}

A partir de 1940 o número de revistas progrediu. As décadas de 1990 e de 2000 tiveram maior fundação de revistas (Figura 1). A soma das revistas fundadas a partir de 2000 até 2014 (15 anos) ultrapassou o século passado (Figura 1). Atualmente a área de Ensino cresce, apesar do recuo aparente da década de 2010. Contudo, não está contabilizado e finalizado a quantidade de revistas fundadas, pois analisamos até o ano de 2014 , porém, ainda que o crescimento seja menor, ainda há uma taxa de crescimento.

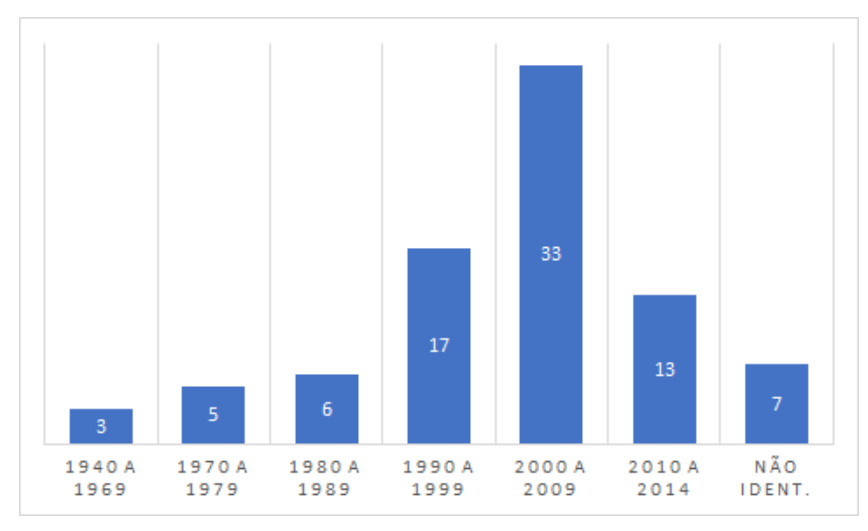

Figura 1: Período de fundação dos periódicos de Ensino Fonte: Os autores.

\subsection{Publicação on-line}

A comunicação científica mudou a abordagem tradicional da revista impressa para a revista digital (on-line) na Internet (Figura 2). A tendência geral das revistas não foi somente a oferta de números recentes na opção virtual, algumas virtualizam seus números anteriores. Desta forma, no portal da CAPES é possível encontrar revistas publicadas desde da década de 1960. 


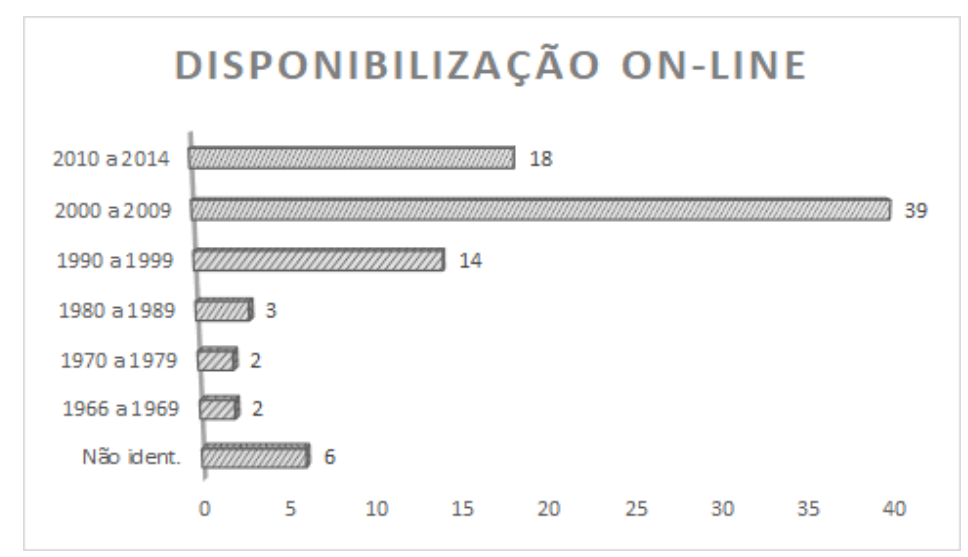

Figura 2: Disponibilização das publicações on-line presentes dos periódicos de Ensino Fonte: Os autores.

\subsection{Frequência de publicação}

A frequência de publicação de novos números relaciona-se à consolidação de produção e demanda desse conteúdo nos periódicos científicos. Encontramos que a periodicidade das revistas foi de publicação quadrimestral e semestral (Quadro 1).

\begin{tabular}{|c|c|c|c|c|c|}
\hline Qualis & Mensal & Bim. & Trim. & Quad. & Sem. \\
\hline A1 & 3 & 1 & 1 & 5 & 3 \\
\hline A2 & 2 & 1 & 6 & 13 & 6 \\
\hline B1 & 0 & 2 & 5 & 12 & 22 \\
\hline Total & 5 & 4 & 12 & 30 & 31 \\
\hline
\end{tabular}

Quadro 1: Frequência de publicação on-line presentes dos periódicos de Ensino

Fonte: Os autores

Atualmente, as revistas com maior frequência são as internacionais, com alguns títulos com periodicidade mensal, bimestral e trimestral, além das quadrimestrais e semestrais. As brasileiras são predominantemente quadrimestrais e semestrais, com poucas bimestrais e trimestrais e nenhuma mensal.

Desta forma, isso se associa com o público que produz e que se relaciona com essas publicações. No caso das revistas brasileiras, de forma geral, são vinculadas aos programas de pós-graduação de diferentes instituições de ensino superior e, sua publicação de "certa forma" é dirigida para a comunidade acadêmica (local) e, depois de consolidado o espaço local do periódico, tende a avançar para os níveis regionais e/ou internacionais. 
Esta questão de demanda e periodicidade pode estar relacionada às instituições que editam os periódicos. É importante ressaltar que esses periódicos nacionais são de acesso gratuito e na sua maioria estão vinculados às instituições de ensino superior, e normalmente dirigidos por programas de pós-graduação na área de conhecimento. Isso pode estar atrelado ao fato de a manutenção desses periódicos serem realizadas por profissionais desses programas, e com a atividade editorial não ser necessariamente remunerada, e, portanto, com o tempo limitado. Desta forma, não há dedicação exclusiva à função, desfavorecendo uma frequência maior naquilo que compete aos números publicados.

Por outro lado, há periódicos que se declaram editados por instituições não universitárias, como segue: Educação e Matemática - Revista da Associação de Professores, Genética na Escola, Natureza \& Conservação, Revista Brasileira de História da Ciência, Revista Brasileira de História da Matemática, Revista de Ensino de Bioquímica, Tendências em Matemática Aplicada e Computacional, Ambiente \& Sociedade, Revista Brasileira de Ensino de Ciência e Tecnologia, Revista Zetetiké e Saúde em Debate. Estes periódicos podem ser observados no Apêndice A.

As revistas internacionais seguem uma tendência diversa, a sua maioria é editada por editoras, associações, centro de pesquisa e instituições não universitárias. Isso talvez possibilite a maior frequência de publicação (mensal, bimestral e outras) devido a possuir corpo próprio de associados de pesquisadores e editores. Neste sentido, muitas dessas revistas são pagas, ou seja, o seu conteúdo se torna de acesso restrito, ou seja, por filiação, seja por meio de assinatura pessoal ou institucional ${ }^{10}$.

Neste sentido é importante o serviço prestado pela CAPES, no programa de acesso à informação científica por meio do Portal de Periódicos da CAPES, por meio do qual muitas revistas são disponibilizadas pelo Governo Federal brasileiro, sem custo aos usuários cadastrados, que têm convênio com os periódicos internacionais de acesso restrito.

\footnotetext{
${ }^{10}$ A International Journal of Science Education associada a editora científica Taylor \& Francis on-line possui a assinatura anual de $\$ 1.558,00$ (USD). Ou pode-se pagar artigos unitariamente com preços variados. Sendo esse tema de um grande debate internacional sobre os valores dos artigos, e sua manipulação lucrativas em que exclui muitos pesquisadores de países em desenvolvimento.
} 


\subsection{Frequência de bases de dados indexados dos periódicos}

Os catálogos de indexação são importantes para armazenamento e distribuição dos números das revistas, bem como para busca de tema, descritor ou palavra-chave em pesquisa. Nas revistas com estrato no Qualis A devem ocorrer a indexação a um repositório científico, da universidade, centro de pesquisa ou órgão que faça o serviço.

A maioria das revistas declaram que estão cadastradas em pelo menos dois catálogos de indexação (Quadro 2). Salientamos que algumas revistas não informaram sua indexação e, portanto, não foi possível quantificá-los.

\begin{tabular}{|c|c|c|c|}
\hline Qualis & Não identi. & 2 a 4 & mais de 5 \\
\hline A1 & 6 & 4 & 3 \\
\hline A2 & 5 & 16 & 9 \\
\hline B1 & 10 & 19 & 12 \\
\hline Total & 21 & 39 & 24 \\
\hline
\end{tabular}

Quadro 2: Frequência de base de dados indexadores de publicação on-line presentes dos periódicos de Ensino

Fonte: Os autores.

A lógica da indexação é que quanto mais as revistas aparecem em catálogos diferentes, maior será seu acesso e consulta. Em especial os periódicos brasileiros que são gratuitos e buscam alcance internacional por meio do sistema de informação. Contudo, publicar em revista que tem maior abertura na indexação não é sinônimo de impacto na comunidade científica, ou de citação de trabalho, mas de melhor infraestrutura para a disponibilização dos conteúdos dos que publicam.

As revistas com indexadores não identificados na pesquisa foram consideradas, "zero" indexadores. Apesar de possivelmente ocorrerem indexações para fazerem parte desta classificação. Os que possuíam mais de oito indexadores, o formulário utilizado para a pesquisa não permitiu o preenchimento, desta forma consideramos o número "dez".

\subsection{Citação em revistas Qualis A1}

Nesta seção, e na seção 2.7 e 2.8 foram realizadas comparações entre dados dos periódicos Qualis em relação às revistas de origem nacional e internacional, quantidade de indexadores visíveis e citações encontradas. 
Os dados de indexadores e origem da revista foram consultados a área de capa das revistas e informações da revista (normalmente presente na aba com a terminação "Sobre").

Sobre as citações foi um desafio definir um procedimento viável em razão das diferentes plataformas de indexação, termos não homogêneos e ferramentas de busca. Desta forma, foi feito a consulta no Google Acadêmico (Scholar Google) no usuário anônimo utilizando-se do nome ou sigla do periódico. Consultamos até a terceira página de resultados da busca e, procuramos os trabalhos mais citados, e com esse procedimento registramos os três trabalhos com maior quantidade de citações.

Entendemos que esse procedimento não é parâmetro definitivo devido às atualizações constantes de citações, e por influência do algoritmo de busca possivelmente privilegiar informações por localidade e idioma. Contudo, foi uma forma possível para construir dados a nível de indicador, e não como resultado descritivo com alta fidedignidade.

No Quadro 3 relacionamos as citações no Google Acadêmico com a quantidade de bases indexadoras e citações. Operamos por média, de modo ao comparar os periódicos Qualis A1 nacionais e internacionais.

\begin{tabular}{|l|c|c|c|c|c|}
\hline \multirow{2}{*}{ Qualis A1 } & \multirow{2}{*}{ N } & \multicolumn{2}{|c|}{ Soma } & \multicolumn{2}{c|}{ Média } \\
\cline { 3 - 6 } & & Indexadores & Citações & Indexadores & Citações \\
\hline Nacional & 3 & 12 & 1.292 & 4,0 & 430,5 \\
\hline Internacional & 10 & 25 & 15.808 & 2,7 & $1.756,5$ \\
\hline
\end{tabular}

Quadro 3: Comparação entre periódicos Qualis A1 de origem nacional e internacional em função da soma e média de indexadores e citações

Fonte: Os autores.

Nesse estrato de classificação têm-se as melhores revistas científicas de ensino. Separamos somente os periódicos de ensino de ciências e/ou matemática. Ocorrendo uma proporção próximo de 3:1 de revistas internacionais para as nacionais. Todavia, mesmo ocorrendo uma média maior de indexadores para os periódicos nacionais, apresenta-se uma diferença de 4:1 em relação a quantidade de citação dos periódicos internacionais para os nacionais.

Isso pode ser explicado pelas políticas dos periódicos nacionais Qualis A1 serem publicadas quadrimestralmente, e os internacionais com periodicidade menor ofertando mais números, a saber: 3 revistas mensais, 1 bimestral, 1 trimestral, 2 quadrimestrais e 3 
semestrais. Por outro lado, o fato de serem publicados predominantemente na língua inglesa e castelhano/espanhola aumenta sensivelmente o universo de leitores, em relação às revistas brasileiras que publicam majoritariamente em língua portuguesa. Logo, mesmo que os trabalhos publicados nas revistas brasileiras sejam de nível internacional, o leitor (pesquisador) necessitará traduzir os textos para realizar citações.

Nesse estrato convém relacionar que são pesquisadores de alto nível que se elevam junto com a revista em razão da qualidade, prestígio e confiança das publicações. Como, por exemplo, na revista "Ensaio: Pesquisa em Educação Em Ciências" com mais de 800 citações em três trabalhos publicados e assinados por Auler, Delizoicov, Mortimer, Lorenzetti, entre outros. Também presente neste estrato o periódico importante como International Journal of Science Education (IJSE) com com mais 4.600 citações em três publicações, assinada por D.F. Treagust, J. Osborne, R. Duit, S. Hodson entre outros.

\subsection{Citação em revistas Qualis A2}

Analisamos as citações no Google Acadêmico com a quantidade de bases indexadoras e citações e, operamos por média para comparar os periódicos Qualis A2 nacionais e internacionais (Quadro 4).

\begin{tabular}{|l|c|c|c|c|c|}
\hline \multirow{2}{*}{ Qualis A2 } & \multirow{2}{*}{ N } & \multicolumn{2}{|c|}{ Soma } & \multicolumn{2}{c|}{ Média } \\
\cline { 3 - 6 } & & Indexadores & Citações & Indexadores & Citações \\
\hline Nacional & 20 & 78 & 4.716 & 4,0 & 236 \\
\hline Internacional & 8 & 43 & 6.239 & 5,5 & 780 \\
\hline
\end{tabular}

Quadro 4: Comparação entre periódicos Qualis A2 de origem nacional e internacional em função da soma e média de indexadores e citações

Fonte: Os autores.

No Quadro 4 verificamos que se inverte a proporção de periódicos, sendo de 2,5:1 das revistas nacionais para as internacionais. Também inverte-se a proporção de indexadores para uma maior quantidade aos periódicos internacionais. Contudo, observa-se que o somatório de citações ainda é superior nos periódicos internacionais, e os valores da média, a proporção de citação supera de 3:1 para as revistas internacionais.

Nestes valores ocorreram disparidades devido à presença de um periódico internacional que superou 4.700 citações (Public Understanding of Science), elevando 
sozinho esse periódico à média com menor número de revistas neste grupo. O periódico nacional com maior número de citações nesse estrato foi de 1.460 citações (Investigações em Ensino de Ciências). Entretanto temos outros periódicos brasileiros com frequência de citação muito baixa.

Cabe aqui chamar atenção, que nas revistas A2, os artigos foram mais citados que nas revistas A1. Logo, em função de comunicação entre pesquisadores é importante não desprezar as revistas de estrato menor.

Ademais, ocorreram seis periódicos nacionais com mais de 200 citações, cinco periódicos com somatório de citações entre 199 a 100, e nove com somatório menor que 100 citações.

\subsection{Citação em revistas Qualis B1}

Verificamos as citações no Google Acadêmico com a quantidade de bases indexadoras e citações e, operamos por média para comparar os periódicos Qualis B1 nacionais e internacionais (Quadro 5).

\begin{tabular}{|l|c|c|c|c|c|}
\hline \multirow{2}{*}{ Qualis B1 } & \multirow{2}{*}{$\mathbf{N}$} & \multicolumn{2}{|c|}{ Soma } & \multicolumn{2}{c|}{ Média } \\
\cline { 3 - 6 } & & Indexadores & Citações & Indexadores & Citações \\
\hline Nacional & 28 & 124 & 3.147 & 4,5 & 112,5 \\
\hline Internacional & 13 & 44 & 1.890 & 3,5 & 145,5 \\
\hline
\end{tabular}

Quadro 5: Comparação entre periódicos Qualis B1 de origem nacional e internacional em função da soma e média de indexadores e citações

Fonte: Os autores.

Nas revistas qualificadas com B1 é possível encontrar a maior quantidade de periódicos, com 41 títulos. A proporção de periódicos é de 2:1 aos periódicos nacionais para os internacionais. Mas o distanciamento de proporção de citações é menor, ainda com vantagem para os periódicos internacionais. Os periódicos nacionais mantêm quantidade de indexadores maior que os internacionais, mas isso não tem reflexo direto na quantidade de citações.

Nesse estrato destaca-se a revista brasileira "Ciência \& Ensino" com 591 citações em três publicações, e depois a revista internacional "Revista Portuguesa de Ciências do Desporto" com 560 citações em três trabalhos publicados.

Contudo, chamamos a atenção que apenas sete revistas nacionais e sete revistas internacionais somam mais de 100 citações, de acordo com o nosso procedimento. 
Logo, há periódicos que não são necessariamente recentes, e contam pouquíssima citações, mesmo que atualmente gozem de espaço virtual (on-line). Neste sentido há necessidade de o pesquisador analisar a viabilidade de submissão para a aceitação na comunidade acadêmica e, de pesquisadores nas temáticas específicas em que se busca comunicar o seu manuscrito.

\section{Considerações finais}

A oportunidade de realização de pesquisa coletiva com utilização de tecnologia de arquivos colaborativos permitiu uma experiência rica de coleta, análise e discussão dos dados construídos sobre os periódicos selecionados da área de ensino. Isto é, enquanto formação educativa nesta disciplina do curso de doutorado. Entendemos a necessidade e dificuldade de trabalharmos juntos com a polifonia e polissemia nos termos e dados registrados, e nisto o esforço de interpretação individual e a socialização para a construção de uma interpretação coletiva foi uma práxis fundamental para compreensão desta realidade que é área de ensino.

Podemos destacar como resultados relevantes sobre os periódicos selecionados da área de ensino: a maior quantidade de periódicos em Ciências do que Matemática; a quantidade não tão distante entre periódicos A2 e B1; destacar que a partir da década de 2000 ocorreu a grande fundação de periódicos e sua disponibilização on-line; os periódicos nacionais são normalmente de periodicidade quadrimestral ou semestral, e geralmente administrados por instituições de ensino superior; os periódicos nacionais normalmente possuem mais de duas bases de indexação, ou mais, todavia isso não necessariamente os tornam mais citados.

As revistas qualificadas em A1 são de maioria internacional e publicam com mais periodicidades que as nacionais. O seu somatório de citação supera a proporção de 4:1 em relação aos periódicos nacionais. Encontram-se nestes periódicos o nome de pesquisadores reconhecidos na área de Educação em Ciências e Matemática.

As revistas qualificadas em A2 são, em sua maioria, de origem nacional, contudo publicam com periodicidade menor e sua proporção de citação é menor que os periódicos internacionais. Entretanto, se apresentaram periódicos com somatórios de citação maiores que os periódicos A1.

As revistas qualificadas em B1 são semelhantes às características dos periódicos A2. Todavia, com somatórios de citação e periodicidade mais modestos. Neste estrato 
registramos revistas sem citações encontradas, ou com somatório baixo comparado os periódicos A2.

Entendemos pertinente recomendar: a) que os escopos das revistas busquem apresentar com clareza sobre os tipos de temáticas e manuscritos que a revista recebe; b) os dados de escopo e indexadores sejam publicados com facilidade de acesso ao leitor/pesquisador; c) a possível disponibilização (virtualização) das publicações impressas e mais antigas facilitam a busca na mídia virtual com acesso da internet; d) a presença e atualização do histórico do periódico auxilia a compreender o escopo e a conhecer outros nomes e, outros possíveis registros de ISSN, e assim facilita reunir a produção da revista; e) muitas revistas internacionais solicitam o resumo em mais de idioma estrangeiro para facilitar o acesso e indexação, pode ser interessante essa atualização em normas dos periódicos brasileiros para atrair mais acessos; f) a possível a publicação dos artigos em outra língua pode ser outra forma de atualização de normas dos periódicos nacionais para superar a barreira idiomática; g) a associação/convênio das equipes editoriais normalmente de instituições de ensino superior com outros grupos competentes (associações, centro de pesquisa, etc.) pode facilitar o diálogo para criação de novidades, divulgação e revisão da estrutura dos periódicos buscando a melhor qualidade e, adequação de alcance aos públicos destinados.

\section{Referências}

BARATA, R. C. B. Dez coisas que você deveria saber sobre o Qualis. Revista Brasileira dePós-Graduação, Brasília, v. 13, n. 30, p. 13-40, 2016. Disponível em: <http://ojs.rbpg.capes.gov.br/index.php/rbpg/article/view/947/pdf>. Acesso em: 18dez. 2017.

BUENO, W. C. Comunicação científica e divulgação científica: aproximações e rupturasconceituais. Informação \& Informação, Brasília, v. 15, n. supl, p. 1-12, 2010.Disponível em:<http://www.uel.br/revistas/uel/index.php/informacao/article/view/6585>. Acesso em:18 dez.2017.

FERREIRA, M. C. G.; KRZYZANOWSKI, R. F. Periódicos científicos: critérios dequalidade. Pesquisa Odontologia do Brasil, São Paulo, v. 17, n. suplem, p. 43-48, 2003. Disponível em: <http://www.scielo.br/pdf/pob/v17s1/a07v17s1.pdf>. Acesso em: 14 ago. 2017.

MALOZZE, G. L. M. Produção científica: periódicos. In: WITTER, G. P. (Org.). Produção científica em psicologia e educação. Campinas: Alínea, 1999. p. 103-122.

MACHADO, A. M. N.; JESUS, P. C. P. G. A "carta periódica": um modelo de publicação interativo para superar o produtivismo acadêmico e qualificar a produção científica. MACAU (CHINA): [s.n.], 2012. p. 1-11.

NASSI-CALÒ, L. Estudo destaca os modelos de publicação em periódicos científicos do Brasil e Espanha [online]. SciELO em Perspectiva, São Paulo, 2014. Disponível 


\section{Vye:BEEM $\mid \begin{gathered}\text { Revista Brasileira de Educação em } \\ \text { Ciências e Educação Matemática }\end{gathered}$ \\ ISSN 2594-9179}

em:<http://blog.scielo.org/blog/2014/04/25/estudo-destaca-os-modelos-de-publicacaoemperiodicos-cientificos-do-brasil-e-espanha/>. Acesso em: 18 dez. 2017.

Recebido em: 27 de abril de 2018.

Aceito em: 30 de abril de 2018. 


\section{APÊNDICE}

Periódicos Qualis Aem que foi verificado no escopo os termos "Educação em Ciências" e/ou

"Educação Matemática".

\begin{tabular}{|c|c|c|c|c|c|c|}
\hline Qualis & Origem & ISSN & Nome do periódico & Period. & Ênfase & Instituição vinculadora \\
\hline $\mathrm{A} 1$ & INT & $1043-4046$ & $\begin{array}{l}\text { Advances in Physiology } \\
\text { Education }\end{array}$ & Mensal & Ciê. & American Physiological Society \\
\hline $\mathrm{A} 1$ & NAC & $1809-4422$ & Ambiente \& Sociedade & Quad. & Ciê. & $\begin{array}{l}\text { Associaçãa Nacional de Pós- } \\
\text { Graduação e Pesquisa em Ambiente e } \\
\text { Sociedade }\end{array}$ \\
\hline $\mathrm{A} 1$ & NAC & $\begin{array}{l}\text { 1036-36X } \\
19804415\end{array}$ & $\begin{array}{l}\text { Bolema. Boletim De } \\
\text { Educação Matemática }\end{array}$ & Quad. & Mat. & UNESP Rio Claro \\
\hline A1 & INT & 0013-1954 & $\begin{array}{l}\text { Educational Studies in } \\
\text { Mathematics }\end{array}$ & Quad. & Mat. & Springer Netherlands \\
\hline $\mathrm{A} 1$ & NAC & $\begin{array}{l}1415-2150 \\
1983-2117\end{array}$ & $\begin{array}{l}\text { Ensaio: Pesquisa em } \\
\text { Educação Em Ciências }\end{array}$ & Quad. & Ciê. & $\begin{array}{l}\text { UFMG - Programa de Pós Graduação } \\
\text { em Educação }\end{array}$ \\
\hline $\mathrm{A} 1$ & INT & $0228-0671$ & $\begin{array}{l}\text { For the Learning of } \\
\text { Mathematics }\end{array}$ & Trim. & Mat. & $\begin{array}{l}\text { University of New Brunswick, } \\
\text { (Canadá), publicado sob o Canadian } \\
\text { Study Mathematical Education Study }\end{array}$ \\
\hline $\mathrm{A} 1$ & INT & 0020-739X & $\begin{array}{l}\text { International Journal of } \\
\text { Mathematical Education in } \\
\text { Science and Technology }\end{array}$ & Bim. & Mat. & Taylor e Francis \\
\hline $\mathrm{A} 1$ & INT & $1571-0068$ & $\begin{array}{l}\text { International Journal of } \\
\text { Science and Mathematical } \\
\text { Education }\end{array}$ & Mensal & Mat. & Springer Link \\
\hline A1 & INT & $0950-0693$ & $\begin{array}{l}\text { International Journal of } \\
\text { Science Education }\end{array}$ & Mensal & Ciê. & Taylor e Francis Online \\
\hline $\mathrm{A} 1$ & INT & $2469-9896$ & $\begin{array}{l}\text { Physical Review Special } \\
\text { Topics. Physics Education } \\
\text { Research }\end{array}$ & Sem. & Ciê. & American Physical Society \\
\hline $\mathrm{A} 1$ & INT & $0031-9120$ & $\begin{array}{l}\text { Physics Education (Bristol. } \\
\text { Print) }\end{array}$ & Sem. & Ciê. & Não identificado \\
\hline $\mathrm{A} 1$ & INT & $1697-011 X$ & $\begin{array}{l}\text { Revista Eureka Sobre } \\
\text { Enseñanza y Divulgación } \\
\text { de las Ciencias }\end{array}$ & Quad. & Ciê. & UCA - Universidade de Cádiz \\
\hline $\mathrm{A} 1$ & INT & $\begin{array}{l}1863-9690 \\
1863-9704\end{array}$ & ZDM (Berlin. Print) & Sem. & Mat. & Não identificado \\
\hline
\end{tabular}


Periódicos Qualis A2 em Educação em Ciências, Educação Matemática e Educação em Ciências e Educação Matemática.

\begin{tabular}{|c|c|c|c|c|c|c|}
\hline Qualis & Origem & ISSN & Nome Do Periódico & Period. & Ênfase & Instituição vinculadora \\
\hline A2 & NAC & $\begin{array}{l}2178- \\
7727 / 1517- \\
4492\end{array}$ & $\begin{array}{l}\text { Acta Scientiae: Revista de } \\
\text { Ensino de Ciências e } \\
\text { Matemática }\end{array}$ & Bim. & $\mathrm{C} \& \mathrm{M}$ & $\begin{array}{l}\text { Universidade Luterana do Brasil sob a } \\
\text { responsabilidade do Programa de Pós- } \\
\text { Graduação em Ensino de Ciências e } \\
\text { Matemática. }\end{array}$ \\
\hline A2 & NAC & $1982-5153$ & Alexandria & Sem. & $\mathrm{C} \& \mathrm{M}$ & UFSC \\
\hline A2 & NAC & $\begin{array}{l}2317-5125 / \\
1980-5128\end{array}$ & $\begin{array}{l}\text { Amazônia - Revista de } \\
\text { Educação em Ciências e } \\
\text { Matemáticas (Online) }\end{array}$ & Sem. & $\mathrm{C} \& \mathrm{M}$ & $\begin{array}{l}\text { Programa de Pós-Graduação em } \\
\text { Educação em Ciências e Matemáticas } \\
\text { do Instituto de Educação Matemática e } \\
\text { Científica - IEMCI - da Universidade } \\
\text { Federal do Pará, Belém/Pará/Brasil. }\end{array}$ \\
\hline A2 & NAC & $\begin{array}{l}0104-0405 / \\
1982-4866\end{array}$ & Dynamis & Sem. & $\mathrm{C} \& \mathrm{M}$ & FURB Blumenau \\
\hline A2 & NAC & $\begin{array}{l}1517-3941 / \\
2317-904 X\end{array}$ & $\begin{array}{l}\text { Educação Matemática em } \\
\text { Revista }\end{array}$ & Trim. & Mat. & Não identificado \\
\hline A2 & NAC & $1518-8221$ & $\begin{array}{l}\text { Educação Matemática em } \\
\text { Revista-RS }\end{array}$ & Quad & Mat. & $\begin{array}{l}\text { Universidade Luterana do Brasil - } \\
\text { ULBRA }\end{array}$ \\
\hline A2 & NAC & $\begin{array}{l}1516-5388 / \\
1983-3156\end{array}$ & $\begin{array}{l}\text { Educação Matemática } \\
\text { Pesquisa }\end{array}$ & Quad & Mat. & $\begin{array}{l}\text { Programa de Estudos Pós-Graduados } \\
\text { em Educação Matemática da PUC-SP, }\end{array}$ \\
\hline A2 & INT & $\begin{array}{l}1132-9157 / \\
23853483\end{array}$ & $\begin{array}{l}\text { Enseñanza de las Ciencias } \\
\text { de la Tierra }\end{array}$ & Quad & Ciê. & Universitat de Girona \\
\hline A2 & NAC & $\begin{array}{l}1518-9384 / \\
1518-8795\end{array}$ & $\begin{array}{l}\text { Investigações Em Ensino } \\
\text { De Ciências }\end{array}$ & Quad & Ciê. & UFRGS \\
\hline A2 & INT & $2176-5634$ & $\begin{array}{l}\text { Jornal Internacional de } \\
\text { Estudos em Educação } \\
\text { Matemática }\end{array}$ & Quad & Mat. & UNIBAN Kroton \\
\hline A2 & INT & $1887-3987$ & $\begin{array}{l}\text { PNA: Revista de } \\
\text { Investigación en Didáctica } \\
\text { de la Matemática }\end{array}$ & Trim & Mat. & $\begin{array}{l}\text { Universidad de Granada. Departamento } \\
\text { de Didáctica da Matemática }\end{array}$ \\
\hline A2 & NAC & $\begin{array}{l}2176-9230 / \\
1661-8157\end{array}$ & Praxis & Quad & Ciê. & $\begin{array}{l}\text { Centro Universitário de Volta Redonda } \\
\text { (UNIFOA) }\end{array}$ \\
\hline A2 & INT & $0963-6625$ & $\begin{array}{l}\text { Public Understanding of } \\
\text { Science }\end{array}$ & Men. & Ciê. & SAGE Journals \\
\hline A2 & INT & 2014-3621 & $\begin{array}{l}\text { REDIMAT - Journal of } \\
\text { Research in Mathematics } \\
\text { Education } \\
\text { REDIMAT- Revista de } \\
\text { Investigación en Didáctica } \\
\text { de las Matemáticas }\end{array}$ & Quad & Mat. & Hipatia Press \\
\hline A2 & INT & $1579-1513$ & $\begin{array}{l}\text { REEC. Revista Electrónica } \\
\text { de Enseñanza de las } \\
\text { Ciencias }\end{array}$ & Trim & Ciê. & Educacion Editora \\
\hline
\end{tabular}




\begin{tabular}{|c|c|c|c|c|c|c|}
\hline A2 & INT & $\begin{array}{l}1479-4802 / \\
1754-0178\end{array}$ & $\begin{array}{l}\text { Research in Mathematics } \\
\text { Education }\end{array}$ & Men. & Mat. & Taylor \& Francis \\
\hline A2 & NAC & $1981-1322$ & $\begin{array}{l}\text { REVEMAT: Revista } \\
\text { Eletrônica de Educação } \\
\text { Matemática }\end{array}$ & Sem. & Mat. & $\begin{array}{l}\text { UFSC/MTM/PPGECT, Florianopolis, } \\
\text { SC. }\end{array}$ \\
\hline A2 & NAC & $1982-873 X$ & $\begin{array}{l}\text { Revista Brasileira de } \\
\text { Ensino de Ciência e } \\
\text { Tecnologia }\end{array}$ & Quad & Ciê. & $\begin{array}{l}\text { Pós-graduação em Ensino de Ciência e } \\
\text { Tecnologia da Universidade } \\
\text { Tecnológica Federal do Paraná - } \\
\text { PPGECT/UTFPR }\end{array}$ \\
\hline A2 & NAC & $\begin{array}{l}1806-5104 / \\
1984-2686\end{array}$ & $\begin{array}{l}\text { Revista Brasileira de } \\
\text { Pesquisa em Educação em } \\
\text { Ciências }\end{array}$ & Quad & Ciê. & $\begin{array}{l}\text { Associação Brasileira de Pesquisa em } \\
\text { Educação em Ciências (ABRAPEC) }\end{array}$ \\
\hline A2 & NAC & $2238-2380$ & $\begin{array}{l}\text { Revista e Educação, } \\
\text { Ciências e Matemática }\end{array}$ & Quad & $\mathrm{C} \& \mathrm{M}$ & $\begin{array}{l}\text { Programa de Pós-Graduação em Ensino } \\
\text { das Ciências na Educação Básica da } \\
\text { Unigranrio }\end{array}$ \\
\hline A2 & NAC & $2179-426 X$ & $\begin{array}{l}\text { Revista de Ensino de } \\
\text { Ciências e Matemática } \\
\text { (RENCIMA) }\end{array}$ & Trim & $\mathrm{C} \& \mathrm{M}$ & $\begin{array}{l}\text { Programa de Pós-Graduação em Ensino } \\
\text { de Ciências e Matemática da } \\
\text { Universidade Cruzeiro do Sul }\end{array}$ \\
\hline A2 & INT & $1850-6666$ & $\begin{array}{l}\text { Revista Electrónica de } \\
\text { Investigación en } \\
\text { Educación en Ciencias }\end{array}$ & Sem. & Ciê. & $\begin{array}{l}\text { Núcleo de Pesquisa em Educação em } \\
\text { Ciência e Tecnologia do Departamento } \\
\text { de Formação de Professores da } \\
\text { Faculdade de Ciências Exatas. NIECYT } \\
\text { - UNICEN }\end{array}$ \\
\hline A2 & INT & $2007-6819$ & $\begin{array}{l}\text { Revista Latinoamericana } \\
\text { de Investigación en } \\
\text { Matemática Educativa } \\
\text { (RELIME) }\end{array}$ & Quad & Mat. & $\begin{array}{l}\text { Colegio Mexicano de Matemática } \\
\text { Educativa }\end{array}$ \\
\hline A2 & NAC & $\begin{array}{l}1981-7746 / \\
1678-1007\end{array}$ & $\begin{array}{l}\text { Revista Trabalho, } \\
\text { Educação e Saúde }\end{array}$ & Quad & Ciê. & $\begin{array}{l}\text { Escola Politécnica de Saúde Joaquim } \\
\text { Venâncio, da Fundação Oswaldo Cruz }\end{array}$ \\
\hline A2 & NAC & $2176-1744$ & Revista Zetetiké & Quad. & Mat. & $\begin{array}{l}\text { Faculdade de Educação da } \\
\text { Universidade Estadual de Campinas } \\
\text { (em parceria editorial com a UFF) }\end{array}$ \\
\hline A2 & NAC & $\begin{array}{l}2358-2898 / \\
0103-1104\end{array}$ & Saúde Em Debate & Trim & Ciê. & Centro Brasileiro de Estudos de Saúde \\
\hline $\mathrm{A} 2$ & INT & $\begin{array}{l}1133-9853 / \\
2014-4784\end{array}$ & Uno (Barcelona. 1994) & Trim & Mat. & GRAÓ \\
\hline A2 & NAC & $1984-7505$ & $\begin{array}{l}\text { Areté - Revista Amazônica } \\
\text { de Ensino de Ciências }\end{array}$ & Sem. & Ciê. & $\begin{array}{l}\text { Programa de Pós-Graduação em } \\
\text { Educação e Ensino de Ciências da } \\
\text { Universidade do Estado do Amazonas }\end{array}$ \\
\hline
\end{tabular}


Periódicos Qualis B1 em Educação em Ciências, Educação Matemática e Educação em Ciências e Educação Matemática.

\begin{tabular}{|c|c|c|c|c|c|c|}
\hline Qualis & Origem & ISSN & Nome do Periódico & Period. & Ênfase & Instituição vinculadora \\
\hline B1 & NAC & $2316-9451$ & Abakós & Sem. & $\mathrm{C} \& \mathrm{M}$ & $\begin{array}{l}\text { Programas de Pós-graduação em } \\
\text { Informática e de Pós-graduação em } \\
\text { Ensino de Ciências e Matemática da } \\
\text { PUC (Minas) }\end{array}$ \\
\hline B1 & INT & $1133-9837$ & $\begin{array}{l}\text { Alambique: Didáctica de } \\
\text { las Ciencias } \\
\text { Experimentales }\end{array}$ & Trim. & Ciê. & Universidad de La Rioja \\
\hline B1 & INT & $0210-4466$ & $\begin{array}{l}\text { Asclepio. Revista de } \\
\text { Historia de la Medicina y } \\
\text { de la Ciencia }\end{array}$ & Sem. & Ciê. & Instituto de Historia \\
\hline B1 & NAC & $2179-5746$ & Biota Amazônia & Trim. & Ciê. & $\begin{array}{l}\text { Curso de Ciências Biológicas da } \\
\text { Universidade Federal do Amapá }\end{array}$ \\
\hline B1 & NAC & $2357-724 X$ & $\begin{array}{l}\text { Boem: Boletim Online de } \\
\text { Educação Matemática }\end{array}$ & Sem. & Mat. & $\begin{array}{l}\text { Departamento de Matemática } \\
\text { (DMAT) e pelo Programa de Pós- } \\
\text { Graduação em Ensino de Ciências, } \\
\text { Matemática e Tecnologias do } \\
\text { CCT/UDESC }\end{array}$ \\
\hline B1 & NAC & $\begin{array}{l}1980-8631 \\
1414-5111\end{array}$ & Ciência \& Ensino & Sem. & Ciê. & $\begin{array}{l}\text { Instituto Federal de Educação, Ciência } \\
\text { e Tecnologia de São Paulo (Campus } \\
\text { Piracicaba) gerenciada pelo Grupo de } \\
\text { Pesquisa em Contexto de } \\
\text { Aprendizagem, Sociedade e Ensino de } \\
\text { Ciências e Tecnologia. }\end{array}$ \\
\hline B1 & NAC & 1984-154X & Ciência em Tela & Sem. & Ciê. & $\begin{array}{l}\text { Rede de Investigação Divulgação e } \\
\text { Educação em Ciências (RIDEC) da } \\
\text { Universidade Federal do Rio de } \\
\text { Janeiro (UFRJ) }\end{array}$ \\
\hline B1 & INT & $\begin{array}{l}1852-1716 \\
0327-5566\end{array}$ & $\begin{array}{l}\text { Ciencia, Docencia y } \\
\text { Tecnología }\end{array}$ & Sem. & Ciê. & Universidad Nacional de Entre Rios \\
\hline B1 & NAC & $0871-7222$ & $\begin{array}{l}\text { Educação e Matemática - } \\
\text { Revista da Associação de } \\
\text { Professores }\end{array}$ & Bim. & Mat. & $\begin{array}{l}\text { Associação dos Professores de } \\
\text { Matemática }\end{array}$ \\
\hline B1 & NAC & $2177-9309$ & $\begin{array}{l}\text { Em Teia - Revista de } \\
\text { Educação Matemática e } \\
\text { Tecnológica } \\
\text { Iberoamericana }\end{array}$ & Quad. & Mat. & $\begin{array}{l}\text { Programa de Pós-Graduação em } \\
\text { Educação Matemática e Tecnológica - } \\
\text { EDUMATEC do Centro de Educação } \\
\text { da UFPE }\end{array}$ \\
\hline B1 & NAC & $2359-4381$ & Ensino \& Pesquisa & Quad. & Ciê. & $\begin{array}{l}\text { Universidade Estadual do Paraná } \\
\text { (UNESPAR), campus de União da } \\
\text { Vitória e ao Centro de Ciências } \\
\text { Humanas e Educação }\end{array}$ \\
\hline B1 & NAC & $2237-4450$ & $\begin{array}{l}\text { Ensino de Ciências e } \\
\text { Tecnologia em Revista }\end{array}$ & Sem. & Ciê. & $\begin{array}{l}\text { Programa de Pós-Graduação em } \\
\text { Ensino Cientifico e Tecnológico } \\
\text { (PPGEnCT) DA URI }\end{array}$ \\
\hline $\mathrm{B} 1$ & NAC & $2236-2983$ & Exitus & Quad. & Ciê. & $\begin{array}{l}\text { Universidade Federal do Oeste do } \\
\text { Pará/UFOPA }\end{array}$ \\
\hline B1 & NAC & $1982-2413$ & $\begin{array}{l}\text { Experiências em Ensino de } \\
\text { Ciências }\end{array}$ & Quad. & $\mathrm{C} \& \mathrm{M}$ & $\begin{array}{l}\text { Grupo de Ensino do Instituto de Física } \\
\text { da Universidade Federal de Mato } \\
\text { Grosso (UFMT) }\end{array}$ \\
\hline
\end{tabular}




\begin{tabular}{|c|c|c|c|c|c|c|}
\hline B1 & NAC & 1980-3540 & Genética na Escola & Sem. & Ciê. & Sociedade Brasileira de Genética \\
\hline B1 & INT & $2346-4712$ & $\begin{array}{l}\text { Gondola: Ensenanza y } \\
\text { Aprendizaje de las Ciencias }\end{array}$ & Sem. & Ciê. & $\begin{array}{l}\text { Licenciatura em Física da } \\
\text { Universidade Distrital Francisco José } \\
\text { de Caldas }\end{array}$ \\
\hline B1 & NAC & $2159-8118$ & $\begin{array}{l}\text { Journal of Humanistic } \\
\text { Mathematics }\end{array}$ & Sem. & Mat. & NÃO LOCALIZADO \\
\hline B1 & INT & $2151-2612$ & $\begin{array}{l}\text { Jume: Journal of Urban } \\
\text { Mathematics Education }\end{array}$ & Sem. & Mat. & $\begin{array}{l}\text { Unidade de Educação em Matemática } \\
\text { do Departamento de Ensino Médio e } \\
\text { Secundário, Georgia State University }\end{array}$ \\
\hline B1 & INT & $1120-6527$ & La Fisica Nella Scuola & Sem. & Ciê. & $\begin{array}{l}\text { Associazione per l'Insegnamento della } \\
\text { Fisica }\end{array}$ \\
\hline B1 & INT & $1648-939 X$ & Natural Science Education & Quad. & Ciê. & $\begin{array}{l}\text { Scientia Educologica Scientific } \\
\text { Methodological Center }\end{array}$ \\
\hline B1 & NAC & $1679-0073$ & Natureza \& Conservação & Sem. & Ciê. & Fundação Grupo Boticário \\
\hline B1 & NAC & $1984-4891$ & Observatorium & Quad. & Ciê. & $\begin{array}{l}\text { Universidade Federal de Uberlândia } \\
\text { (UFU) }\end{array}$ \\
\hline B1 & NAC & $1982-7652$ & $\begin{array}{l}\text { Perspectivas da Educação } \\
\text { Matemática }\end{array}$ & Quad. & Mat. & $\begin{array}{l}\text { Universidade Federal de Mato Grosso } \\
\text { do Sul }\end{array}$ \\
\hline B1 & NAC & $2177-580 X$ & $\begin{array}{l}\text { Pesquisa em Educação } \\
\text { Ambiental }\end{array}$ & Sem. & Ciê. & Universidade de São Paulo \\
\hline B1 & NAC & $1984-4352$ & $\begin{array}{l}\text { Pracs: Revista Eletrônica } \\
\text { de Humanidades do Curso } \\
\text { de Ciências Sociais }\end{array}$ & Sem. & Ciê. & Universidade Federal do Amapá \\
\hline B1 & INT & 0872-3915 & Quadrante (Lisboa) & Sem. & Mat. & $\begin{array}{l}\text { Associação de Professores de } \\
\text { Matemática }\end{array}$ \\
\hline B1 & NAC & $\begin{array}{l}1983-4713 \\
2176-3275\end{array}$ & $\begin{array}{l}\text { Revista Brasileira de } \\
\text { História da Ciência }\end{array}$ & Sem. & Ciê. & $\begin{array}{l}\text { Sociedade Brasileira de História da } \\
\text { Ciência }\end{array}$ \\
\hline B1 & NAC & $1519-955 X$ & $\begin{array}{l}\text { Revista Brasileira de } \\
\text { História da Matemática }\end{array}$ & Sem. & Mat. & $\begin{array}{l}\text { Sociedade Brasileira de História da } \\
\text { Matemática }\end{array}$ \\
\hline B1 & NAC & $\begin{array}{l}1980-220 X \\
0080-6234\end{array}$ & $\begin{array}{l}\text { Revista da Escola de } \\
\text { Enfermagem da USP }\end{array}$ & Bim. & Ciê. & Universidade de São Paulo \\
\hline B1 & NAC & $2238-6629$ & $\begin{array}{l}\text { Revista de Biotecnologia \& } \\
\text { Ciência }\end{array}$ & Sem. & Ciê. & Universidade Estadual de Goiás \\
\hline B1 & INT & 0329-5192 & $\begin{array}{l}\text { Revista de Educación en } \\
\text { Biología }\end{array}$ & Sem. & Ciê. & Universidad Nacional de Córdoba \\
\hline B1 & INT & 0326-7091 & $\begin{array}{l}\text { Revista de Enseñanza de la } \\
\text { Física }\end{array}$ & Sem. & Ciê. & $\begin{array}{l}\text { Asociación de Profesores de Física de } \\
\text { la Argentina }\end{array}$ \\
\hline B1 & NAC & $2318-8790$ & $\begin{array}{l}\text { Revista de Ensino de } \\
\text { Bioquímica }\end{array}$ & Quad. & Ciê. & $\begin{array}{l}\text { Sociedade Brasileira de Bioquímica e } \\
\text { Biologia Molecular }\end{array}$ \\
\hline B1 & NAC & $2447-0783$ & $\begin{array}{l}\text { Revista Eletrônica } \\
\text { Científica Ensino } \\
\text { Interdisciplinar }\end{array}$ & Trim. & Ciê. & $\begin{array}{l}\text { Grupo de Pesquisa Contexto e } \\
\text { Educação (CNPq/UERN), e ao } \\
\text { Programa de Pós-Graduação em } \\
\text { Ensino - POSENSINO (UERN) }\end{array}$ \\
\hline B1 & NAC & 1518-1944 & $\begin{array}{l}\text { Revista Eletrônica de } \\
\text { Enfermagem }\end{array}$ & Quad. & Ciê. & $\begin{array}{l}\text { Faculdade de Enfermagem da } \\
\text { Universidade Federal de Goiás }\end{array}$ \\
\hline
\end{tabular}




\begin{tabular}{|c|c|c|c|c|c|c|}
\hline B1 & NAC & $\begin{array}{l}2179-6955 \\
2236-2150\end{array}$ & $\begin{array}{l}\text { Revista Eletrônica Debates } \\
\text { em Educação Científica e } \\
\text { Tecnológica }\end{array}$ & Trim. & $\mathrm{C} \& \mathrm{M}$ & $\begin{array}{l}\text { Programa de Pós-graduação em } \\
\text { Educação em Ciências e Matemática } \\
\text { Programa de Pós-graduação em } \\
\text { Ensino de Humanidades IFES }\end{array}$ \\
\hline B1 & NAC & $2238-5800$ & $\begin{array}{l}\text { Revista Paranaense de } \\
\text { Educação Matemática }\end{array}$ & Sem. & Mat. & $\begin{array}{l}\text { Curso de Matemática da Universidade } \\
\text { Estadual do Paraná/Câmpus de } \\
\text { Campo Mourão e ao Grupo de } \\
\text { Pesquisa em Educação Matemática de } \\
\text { Campo Mourão - GPEMCAM }\end{array}$ \\
\hline B1 & INT & $1645-0523$ & $\begin{array}{l}\text { Revista Portuguesa de } \\
\text { Ciências do Desporto }\end{array}$ & Quad. & Ciê. & $\begin{array}{l}\text { Faculdade de Desporto da } \\
\text { Universidade do Porto }\end{array}$ \\
\hline B1 & INT & $2077-2327$ & $\begin{array}{l}\text { Science Education } \\
\text { International }\end{array}$ & Quad. & Ciê. & $\begin{array}{l}\text { International Council of Associations } \\
\text { for Ciências Education (ICASE) }\end{array}$ \\
\hline B1 & NAC & $\begin{array}{l}1677-1966 \\
2179-8451\end{array}$ & $\begin{array}{l}\text { Tendências Em Matemática } \\
\text { Aplicada E Computacional }\end{array}$ & Trim. & Mat. & $\begin{array}{l}\text { Brazilian Society of Applied and } \\
\text { Computational Mathematics } \\
\text { (SBMAC) }\end{array}$ \\
\hline B1 & INT & $1815-0640$ & $\begin{array}{l}\text { Unión (San Cristobal de la } \\
\text { Laguna) }\end{array}$ & Quad. & Mat. & $\begin{array}{l}\text { Federação Ibero-Americana de } \\
\text { Educação Matemática (FISEM) }\end{array}$ \\
\hline
\end{tabular}

